\title{
Estrutura da comunidade de macroinvertebrados bentônicos de um riacho de serra em Itatinga, São Paulo, Brasil
}

\author{
Ludmilla O. Ribeiro \& Virginia S. Uieda
}

Departamento de Zoologia, Instituto de Biociências, Universidade Estadual Paulista. Caixa Postal 510, 18618-000 Botucatu, São Paulo, Brasil. E-mail: jbludy@yahoo.com.br; vsuieda@ibb.unesp.br

\begin{abstract}
Structure of a benthic macroinvertebrates community in a mountain stream in Itatinga, São Paulo, Brazil. The benthic macroinvertebrates community of a third order stream was studied during the wet and dry seasons. The community was analyzed using artificial substrates sampled weekly during 56 days in each season. The community composition was characterized by low species diversity, with high number of rare species and few abundant species for both seasons. A high dominance of Chinonomidae (Diptera) for both seasons, followed by Baetidae (Ephemeroptera) and Ancylidae (Mollusca), respectively for the wet and dry seasons, was found. The results reinforced the high importance of Chironomidae and the seasonal effect determining the stream benthic community structure.
\end{abstract}

KEY WORDS. Artificial substrate, aquatic macroinvertebrates, manipulative experiments.

RESUMO. A comunidade de macroinvertebrados bentônicos de um riacho de terceira ordem foi analisada em duas estações do ano, chuvosa e seca. Substratos artificiais foram amostrados semanalmente, ao longo de 56 dias para a coleta de macroinvertebrados, nas duas estações do ano. A composição da comunidade foi caracterizada por uma baixa diversidade, ou seja, presença de muitas espécies raras e poucas espécies abundantes nas duas estações do ano. Uma alta dominância de Chironomidae (Diptera) nas duas estações, seguida de Baetidae (Ephemeroptera) e Ancylidae (Mollusca), respectivamente nas estações chuvosa e seca, foi característico. Os resultados obtidos reforçam a grande importância de Chironomidae na comunidade bentônica de riachos e salientam a influência da sazonalidade sobre a estruturação destes organismos.

PALAVRAS CHAVE. Substrato artificial, macroinvertebrados aquáticos, manipulação experimental.

Os macroinvertebrados de água doce constituem um grupo diversificado de organismos que habitam tanto ambientes lênticos (reservatórios, lagos e lagoas) como lóticos (rios, riachos e córregos) (Hauer \& Resh 1996, Merritt \& Cummins 1996).

A comunidade de macroinvertebrados em ambientes lóticos está representada por vários filos, como Arthropoda (insetos, ácaros, crustáceos), Mollusca (gastrópodos e bivalves), Annelida (oligoquetos), Nematoda e Platyhelminthes (HAUER \& Resh 1996). Desta fauna de invertebrados, os insetos destacam-se em termos de diversidade e abundância (Hynes 1970, LAKE 1990), sendo sua distribuição relacionada às características morfométricas e físico-químicas do habitat, à disponibilidade de recursos alimentares e ao hábito das espécies (RESH \& Rosenberg 1984, BoltovsKoy et al. 1995, MerRitT \& Cummins 1996). Outro fator de grande importância na distribuição dos organismos é a velocidade da correnteza, que pode variar nas diferentes estações do ano. A correnteza pode agir sobre a natureza do substrato, interferindo assim na estrutura das comunidades de invertebrados (WHITTON 1975).

A estrutura da comunidade e o processo de colonização por macroinvertebrados podem ser avaliados em estudos experi- mentais utilizando-se substratos artificiais. O uso destes substratos geralmente resulta numa maior precisão dos dados (LAMBERTI \& RESH 1985), por permitir a padronização da área de amostragem e do tempo inicial do processo de colonização. Os substratos artificiais são um interessante meio de avaliar a dinâmica da comunidade de macroinvertebrados frente às condições ambientais a que são expostos, como forte fluxo da corrente, flutuações anuais ou em curtos intervalos de tempo (RodRígukz et al. 1998).

Neste trabalho foi analisada a composição de macroinvertebrados de um riacho de terceira ordem, em duas estações do ano, a fim de verificar a possível influência da sazonalidade sobre a estrutura desta comunidade.

\section{MATERIAL E MÉTODOS}

\section{Área de estudo}

O trabalho foi realizado no Ribeirão da Quinta (Itatinga, São Paulo), um riacho de terceira ordem que nasce nas escarpas da borda leste da Cuesta de Botucatu, se dirigindo para oeste e desaguando no Ribeirão da Posse, o qual por sua vez deságua no Rio Santo Inácio, afluente do Alto Paranapanema. 
O trecho trabalhado do Ribeirão da Quinta localiza-se no km 204 da Rodovia Castelo Branco (2306'47"S, 48²9'46”W), a $743 \mathrm{~m}$ de altitude e caracteriza-se por apresentar poções, rápidos e corredeiras intercalados ao longo de sua extensão. A área de rápido ("run", segundo Rincón 1999) foi selecionada para este estudo por apresentar características intermediárias entre as de poção e corredeira, permitindo maior homogeneidade entre as amostras e réplicas. Esta área do riacho tem aproximadamente 7 $\mathrm{m}$ de extensão, $4 \mathrm{~m}$ de largura e $10 \mathrm{~cm}$ de profundidade, com fundo arenoso-rochoso, constituído principalmente por rochas de aproximadamente $3 \mathrm{~cm}$ de diâmetro.

\section{Metodologia de amostragem}

O trabalho foi desenvolvido em 2001, durante as estações chuvosa ( 21 de março a 16 de maio) e seca (18 de julho a 5 de setembro). Para a amostragem dos macroinvertebrados a mesma metodologia foi utilizada nas duas estações do ano. Substratos artificiais (placas de cerâmica não vitrificada, de 4x12 $\mathrm{cm}$ ) foram instalados no fundo do rápido e não manuseados por 21 dias. Após este período, os substratos foram amostrados semanalmente, ao longo de 56 dias, para a coleta de macroinvertebrados. Cada cerâmica foi cuidadosamente retirada da água, com auxílio de uma peneira de granulometria $(0,25 \mathrm{~mm})$ para evitar a perda dos organismos. Logo em seguida, a cerâmica foi raspada com o auxílio de uma espátula de metal, sendo o material removido acondicionado em frasco com álcool 70\%, devidamente etiquetado.

No laboratório, o material fixado foi corado com Rosa de Bengala, numa concentração de $12 \mathrm{mg} / \mathrm{l}$, para facilitar a visualização dos macroinvertebrados durante a triagem (MASON \& Yevich 1967). Após 24 horas no corante, este material foi despejado em um conjunto de três peneiras de granulometria (GRANUTEST), com malhas de 1,0; 0,50 e 0,25 mm. As peneiras foram vistoriadas individualmente sob estereomicroscópio, sendo os macroinvertebrados retidos na malha retirados e fixados em álcool $70 \%$. Apesar de, por convenção, o termo macroinvertebrado se referir aos invertebrados que ficam retidos em uma malha de $0,50 \mathrm{~mm}$, no presente trabalho foi adotada a malha de 0,25 mm como limite mínimo para a triagem, como sugerido por Hauer \& Resh (1996), pois muitas larvas de Chironomidae (Diptera) de pequeno diâmetro passam pela malha maior.

Os macroinvertebrados triados foram identificados com auxílio de chaves (Pennak 1978, Lopretto \& Tell 1995, MerritT \& Cummins 1996) e analisados quanto à abundância (número de indivíduos) e área absoluta ocupada $\left(\mathrm{mm}^{2}\right)$. O cálculo da área foi obtido através do mensuramento dos organismos sobre uma placa milimetrada com auxílio de um estereomicroscópio. Esta medida permitiu verificar a importância relativa dos diferentes táxons na ocupação da área do substrato disponibilizada para colonização. A identificação, quando possível, foi realizada até o nível de família, sendo este o menor nível taxonômico utilizado para determinação das UTO's (unidade taxonômica operacional).

Revista Brasileira de Zoologia 22 (3): 613-618, setembro 2005
Em cada dia de coleta também foram tomadas medidas de $\mathrm{pH}$ (pH-metro Corning modelo 35), condutividade (condutivímetro TDS Testr $1^{\mathrm{TM}}$ ), temperatura da água (termômetro de coluna de mercúrio) e velocidade da correnteza (fluxímetro eletrônico Ohio Professional). Os valores de pluviosidade registrados em cada estação de coleta foram obtidos na estação metereológica de Pardinho (São Paulo), localizada a cerca de $1 \mathrm{~km}$ da área de estudo.

\section{Análise dos dados}

Para comparar a abundância e área absoluta ocupada pelas UTO's entre as estações do ano foi utilizado o teste $t$ de Student $(\alpha=0,05)$ (Statistica 1996).

A estrutura da comunidade de macroinvertebrados em cada estação foi analisada pela curva de importância das espécies ou curva de abundância relativa. A representação gráfica dos dados de abundância permite analisar aspectos da comunidade, como por exemplo a diversidade e dominância de espécies (BROWER \& ZAR 1984). Para a obtenção desta curva foi construído um "rank" de espécies, onde a mais abundante é considerada a espécie 1 no "rank", a segunda mais abundante é a espécie 2 no "rank" e assim sucessivamente até a espécie menos abundante.

\section{RESULTADOS}

\section{Parâmetros ambientais}

A estação chuvosa apresentou os menores valores de condutividade $(87 \pm 11,1)$ e $\mathrm{pH}(7,4 \pm 0,3)$, porém temperaturas mais elevadas $(21 \pm 3,0)$ e maior velocidade da correnteza $(0,19 \pm 0,07)$. Por outro lado, na estação seca a condutividade $(131 \pm 89,7)$ e o $\mathrm{pH}(7,9 \pm 0,5)$ foram mais elevados, enquanto a temperatura $(18 \pm 2,1)$ e a correnteza da água $(0,14)$ mostraram valores inferiores aos da estação chuvosa (Tab. I).

O valor de precipitação total na estação chuvosa foi de $141,7 \mathrm{~mm}$ e na estação seca, de 102,9 mm. Embora os valores totais sejam próximos, na estação chuvosa constatou-se nove picos de precipitação pluviométrica, sendo os maiores valores registrados entre o $7^{\circ}$ e $14^{\circ}$ dia e após o $49^{\circ}$ dia de amostragem (Fig. 1). Na estação seca, observaram-se apenas cinco picos de precipitação os quais, apesar de terem ocorrido em intervalos de amostragem semelhantes aos da estação chuvosa, foram de menor intensidade.

\section{Comunidade de macroinvertebrados}

Foram amostrados os seguintes filos: Cnidaria, Platyhelminthes, Nematoda, Mollusca, Annelida e Arthropoda (Tab. II). Dentre estes, o Filo Arthropoda foi o mais representativo, sobressaindo-se a Classe Insecta com sete ordens.

Do total de 32 UTO's amostradas, foram encontradas 26 nas duas estações, no entanto, a abundância total de macroinvertebrados foi maior na estação seca (Tab. II).

Diptera-Chironomidae representou 66\% da abundância total de macroinvertebrados, sendo dominante nas duas estações (Tab. II). Observou-se uma diferença entre as estações chuvosa e seca quanto à seqüência dos grupos dominantes, ocor- 

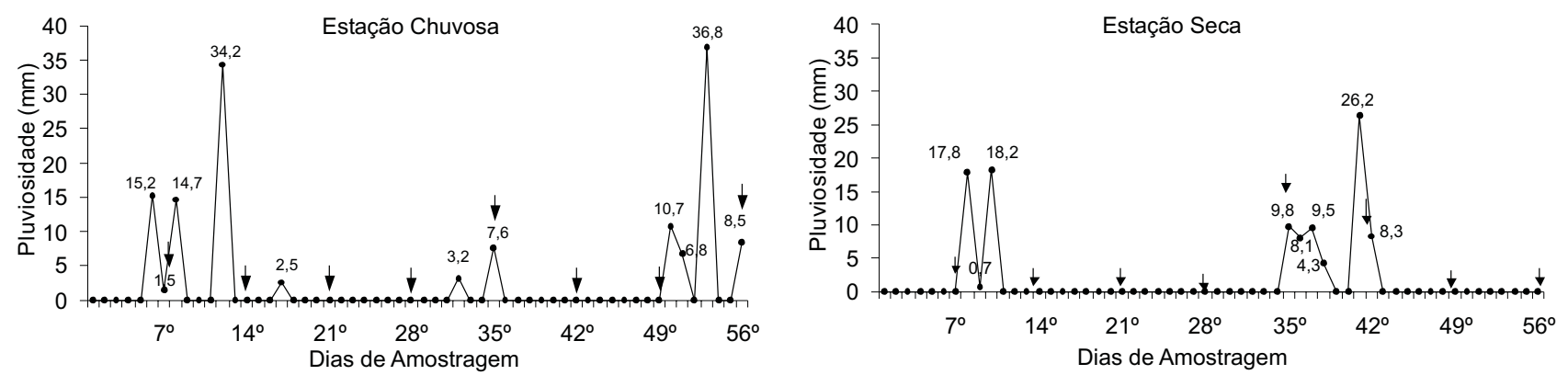

Figura 1. Valores de pluviosidade registrados durante os 56 dias do experimento de colonização, realizado nas estações chuvosa e seca, sendo salientadas (flecha) as datas de amostragem.

Tabela I. Parâmetros físicos e químicos da água analisados durante as estações chuvosa e seca, no Ribeirão da Quinta, e obtidos semanalmente ao longo de 56 dias.

\begin{tabular}{cccccc}
\hline Estações & Dia de amostragem & Condutividade $\left(\mu \mathrm{S} \mathrm{cm}^{-1}\right)$ & $\mathrm{pH}$ & Temperatura da água $\left({ }^{\circ} \mathrm{C}\right)$ & Correnteza $(\mathrm{m} / \mathrm{s})$ \\
\hline Chuvosa & $7^{\circ}$ & 70 & 8,0 & 22,5 & 0,15 \\
& $14^{\circ}$ & 90 & 7,3 & 25,0 & 0,15 \\
& $21^{\circ}$ & - & - & 21,5 & 0,23 \\
& $28^{\circ}$ & 80 & 7,3 & 22,0 & 0,22 \\
& $35^{\circ}$ & 100 & 7,0 & 23,0 & 0,15 \\
& $42^{\circ}$ & 90 & 7,3 & 19,7 & 0,15 \\
& $49^{\circ}$ & 80 & 7,3 & 16,2 & 0,22 \\
\hline Seca & $56^{\circ}$ & 100 & 7,4 & 17,0 & 0,29 \\
& $7^{\circ}$ & 80 & 7,6 & 17,0 & 0,15 \\
& $14^{\circ}$ & 180 & 7,8 & 18,0 & 0,15 \\
& $21^{\circ}$ & 330 & 7,4 & 15,0 & 0,15 \\
& $28^{\circ}$ & 70 & 8,2 & 18,0 & 0,15 \\
& $35^{\circ}$ & 70 & 8,1 & 16,0 & 0,14 \\
& $42^{\circ}$ & 70 & 8,9 & 18,0 & 0,15 \\
& $49^{\circ}$ & 140 & 7,8 & 22,0 & 0,15 \\
\hline $6^{\circ}$ & 110 & 7,6 & 19,0 & 0 \\
\hline
\end{tabular}

rendo uma inversão a partir do segundo lugar entre Ephemeroptera-Baetidae (estação chuvosa) e Mollusca-Ancylidae (estação seca). Além disso, surgiu um terceiro grupo predominante na estação seca, Annelida-Oligochaeta (Tab. II).

Em relação à área ocupada pelas UTO's, a área total ocupada foi maior na estação seca. Quanto aos grupos predominantes, repetiu-se o observado para a abundância, com exceção do terceiro grupo na estação seca, que passou a ser Trichoptera-Hydroptilidae (Tab. II).

Apesar da abundância e área ocupada pelos macroinvertebrados terem sido diferentes quando comparadas as estações, esta diferença não foi significativa $(\mathrm{p}>0,05)$.

Através das curvas de importância de espécies construídas para as duas estações, verificou-se uma baixa diversidade, ou seja, presença de muitas espécies raras e poucas abundantes (Fig. 2), determinada principalmente pela alta dominância de
Chironomidae (espécie 1, Fig. 2), seguida de Baetidae e Ancylidae (espécie 2 , respectivamente para a estação chuvosa e seca).

\section{DISCUSSÃO}

Os fatores físico-químicos são de grande importância na estruturação ecológica dos biótopos aquáticos, determinando a ocorrência e distribuição dos organismos (KLEEREKOPER 1990). Os valores registrados no Ribeirão da Quinta para condutividade, $\mathrm{pH}$ e temperatura refletiram diferenças sazonais, com os maiores valores de condutividade e os menores valores de temperatura tendo sido encontrados na estação seca. Esta estação compreende os meses de inverno, caracterizada por prolongados intervalos de estiagem, o que pode ter influenciado na concentração de íons presentes. KLEEREKOPER (1990), estudando lagoas litorâneas no Rio Grande do Sul, encontrou valores elevados de condutividade depois de prolongado período de 
Tabela II. Abundância total (N) e área ocupada total (Ao) dos taxons amostrados durante as duas estações do ano no Ribeirão da Quinta (estágio juvenil para todos os insetos aquáticos).

\begin{tabular}{|c|c|c|c|c|c|c|}
\hline \multirow{2}{*}{ Grupos taxonômicos (UTO's) } & \multicolumn{2}{|c|}{ Estação chuvosa } & \multicolumn{2}{|c|}{ Estação seca } & \multirow{2}{*}{ N Geral } & \multirow{2}{*}{ Ao Geral } \\
\hline & $\mathrm{N}$ & Ao & $\mathrm{N}$ & Ao & & \\
\hline Cnidaria - Hydrozoa & 1 & 0,2 & - & - & 1 & 0,2 \\
\hline Platyhelminthes - Turbellaria & 3 & 6,7 & 3 & 3,8 & 6 & 10,5 \\
\hline Nematoda & 4 & 1,2 & 1 & 0,2 & 5 & 1,4 \\
\hline Annelida - Oligochaeta & 979 & 358,3 & 1163 & 530,0 & 2142 & 888,3 \\
\hline Mollusca - Gastropoda - Ancylidae & 195 & 229,1 & 2316 & 3375,6 & 2511 & 3604,7 \\
\hline Crustacea - Copepoda & 1 & 0,4 & 54 & 10,7 & 55 & 11,1 \\
\hline Crustacea - Decapoda - Aeglidae & - & - & 1 & 2,5 & 1 & 2,5 \\
\hline Arachnida - Acarina & 9 & 4,1 & 24 & 5,8 & 33 & 9,9 \\
\hline Plecoptera - Família não determinada & - & - & 2 & 0,5 & 2 & 0,5 \\
\hline Plecoptera - Perlidae & 1 & 25,0 & - & - & 1 & 25,0 \\
\hline Ephemeroptera - Família não determinada & 777 & 177,6 & 240 & 308,9 & 1017 & 486,5 \\
\hline Ephemeroptera - Leptohyphidae & 29 & 61,8 & 108 & 143,4 & 137 & 205,2 \\
\hline Ephemeroptera - Leptophlebiidae & 1 & 1,0 & 2 & 1,8 & 3 & 2,8 \\
\hline Ephemeroptera - Baetidae & 2332 & 2379,5 & 20 & 29,7 & 2352 & 2409,2 \\
\hline Odonata - Família não determinada & 4 & 3,8 & 1 & 1,2 & 5 & 5,0 \\
\hline Odonata - Coenagrionidae & 2 & 24,0 & - & - & 2 & 24,0 \\
\hline Odonata - Calopterygidae & 4 & 20,0 & - & - & 4 & 20,0 \\
\hline Trichoptera - Família não determinada & 345 & 92,4 & 563 & 137,3 & 908 & 229,7 \\
\hline Trichoptera - Hydropsychidae & 57 & 342,2 & 9 & 11,9 & 66 & 354,1 \\
\hline Trichoptera - Hydroptilidae & 158 & 261,3 & 757 & 1992,3 & 915 & 2253,6 \\
\hline Trichoptera - Leptoceridae & - & - & 1 & 12,0 & 1 & 12,0 \\
\hline Trichoptera - Glossosomatidae & 107 & 299,8 & 160 & 328,7 & 267 & 628,5 \\
\hline Lepidoptera - Pyralidae & 6 & 22,1 & - & - & 6 & 22,1 \\
\hline Coleoptera - Elmidae & 46 & 14,6 & 18 & 10,3 & 64 & 24,9 \\
\hline Coleoptera - Psephenidae & 10 & 12,0 & 5 & 71,3 & 15 & 83,3 \\
\hline Diptera - Ceratopogonidae & - & - & 1 & 0,1 & 1 & 0,1 \\
\hline Diptera - Chironomidae & 9522 & 3183,6 & 11604 & 4497,5 & 21126 & 7681,1 \\
\hline Diptera - Empididae & 18 & 10,9 & 10 & 3,0 & 28 & 13,9 \\
\hline Diptera - Psychodidae & - & - & 3 & 2,5 & 3 & 2,5 \\
\hline Diptera - Simuliidae & 43 & 40,3 & 16 & 5,1 & 59 & 45,4 \\
\hline Diptera - Tabanidae & - & - & 1 & 3,0 & 1 & 3,0 \\
\hline Número UTO's por estação & 25 & & 26 & & 31 & \\
\hline $\mathrm{N}$ e Ao total por estação & 14654 & 7571,9 & 17083 & 11489,1 & 31737 & 19061,0 \\
\hline
\end{tabular}

seca. Os valores de correnteza também variaram em função da sazonalidade, de modo que os maiores valores na estação chuvosa estão relacionados à maior pluviosidade registrada neste período.

A comunidade de macroinvertebrados no Ribeirão da Quinta esteve representada por todos os grupos taxonômicos comumente citados como representantes da comunidade lótica bentônica. De acordo com Hynes (1970), os insetos compõem a maior parte da fauna de águas correntes, predominando em di- versidade e abundância. No riacho estudado, os insetos dominaram, destacando-se Ephemeroptera (Baetidae), Trichoptera (Hydroptilidae e estágios muito iniciais sem possibilidades de determinação da família) e Diptera (Chironomidae). UiedA \& GAJARDo (1996), analisando a diversidade e distribuição de macroinvertebrados perifíticos no Córrego Itaúna (São Paulo), afluente da mesma Bacia do Ribeirão da Quinta (Bacia do Rio Paranapanema), também constataram um maior predomínio de insetos, dos quais foram mais abundantes Diptera, Plecoptera, 


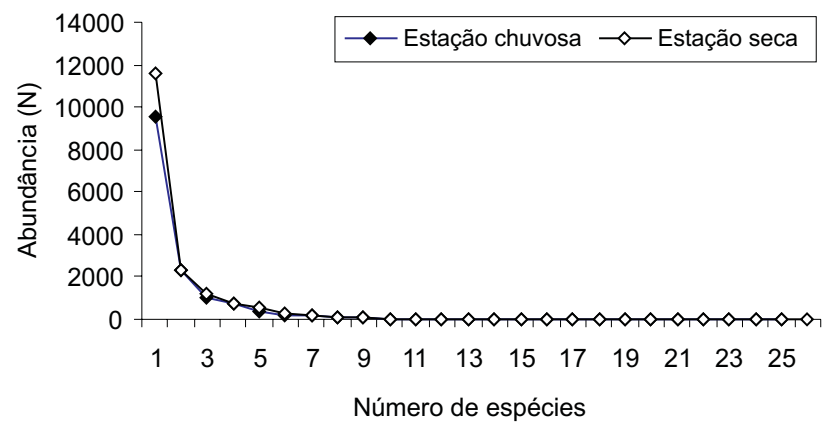

Figura 2. Curva de importância de espécies representando a abundância relativa dos macroinvertebrados amostrados no Ribeirão da Quinta nas estações chuvosa e seca.

Coleoptera e Ephemeroptera. Para a ordem Diptera os autores acima também assinalaram um predomínio de Chironomidae, porém para Ephemeroptera encontraram predomínio de Leptohyphidae ( $=$ Tricorythidae). $\mathrm{O}$ riacho estudado por estes autores apresenta características muito semelhantes ao Ribeirão da Quinta, porém foram amostrados os invertebrados associados ao folhiço acumulado em corredeiras e poções, o que pode ter determinando as diferenças observadas quanto aos grupos predominantes.

A quantidade de macroinvertebrados (abundância e área ocupada) amostrados no Ribeirão da Quinta foi maior na estação seca, apesar da diferença não ter sido significativa, talvez devido à maior estabilidade física nesta estação. Na estação chuvosa, o aumento da correnteza pode provocar o arraste dos organismos rio abaixo. Oliveira et al. (1997), analisando a fauna de Ephemeroptera, Plecoptera e Trichoptera em três riachos do Parque Ecológico de Goiânia (Goiás), verificaram que as maiores alterações na abundância destes grupos foram devidas à pluviosidade, vazão e velocidade da correnteza. KIKUCHI \& UIEDA (1998), estudando a comunidade de macroinvertebrados do Córrego Itaúna, encontraram menor porcentagem de organismos na estação chuvosa, também atribuindo este fato ao aumento da pluviosidade e arraste dos organismos. Huamantinco \& Nessimian (1999) também reportaram maior número de larvas no inverno (período seco), o que poderia estar relacionado à estabilidade ambiental.

A curva de importância de espécies representou a estruturação da comunidade de macroinvertebrados do Ribeirão da Quinta. A grande inclinação do início da curva foi definida pela predominância de Chironomidae durante todo o período de estudo. As larvas de Chironomidae ocorrem normalmente em altas densidades e diversidade, com ciclo de vida curto e biomassa total elevada, o que lhes confere um importante papel no fluxo energético (Coffman \& Ferrington Jr. 1988 apud Higuti et al. 1993). Diversos trabalhos desenvolvidos em riachos no Brasil têm salientado a grande abundância de Chironomidae e sua colonização em uma grande variedade de mesohabitats (e.g. Nessimian 1996, SAnseverino et al. 1998, Serrano et al. 1998, Nessimian et al. 2003).
A dominância de Ephemeroptera-Baetidae na estação chuvosa pode estar relacionada às adaptações morfológicas deste grupo à correnteza. Alguns efemerópteros possuem o corpo achatado, liso e alongado, com pernas que se projetam lateralmente ao corpo, reduzindo o arraste e aumentando a fricção contra o substrato (MERRITT \& CuMmins 1996). Já na estação seca, o segundo grupo dominante foi representado por Mollusca-Ancylidae, o qual parece estar relacionado com a qualidade do habitat, pois este grupo foi predominante no período de maior quantidade de sedimento depositado sobre os substratos artificiais.

Apesar de não ter havido uma diferença estacional significativa na abundância total de macroinvertebrados do Ribeirão da Quinta, provavelmente devido à alta dominância de Chironomidade, estruturalmente as comunidades amostradas nas duas estações diferiram se considerados os grupos seguintes em dominância. Assim, estes resultados não somente reforçam a grande importância de Chironomidae na comunidade bentônica de riachos, mas também salientam a influência de fatores abióticos sobre a estruturação destes organismos.

Dos diversos fatores abióticos de importância na estruturação da comunidade bentônica, a pluviosidade foi o fator que, aparentemente, mais influenciou sua composição, causando um aumento no volume d'água, e assim levando ao arraste dos organismos. Além disto, o aumento da pluviosidade causou alterações nas condições químicas da água, como pH e condutividade, e na quantidade de sedimento, alterando a qualidade do habitat e assim a estrutura da comunidade de macroinvertebrados.

\section{AGRADECIMENTOS}

À CAPES pela bolsa concedida ao primeiro autor durante a realização deste estudo e à FAPESP pelo auxílio financeiro.

\section{REFERÊNCIAS BIBLIOGRÁFICAS}

Boltovskoy, D.; G. Tell \& R. Dadon. 1995.Afinidad entre comunidades bentonicas de un ambiente lotico, p. 203-214. In: E.C. Lopretto \& G.Tell (Eds). Ecosistemas de aguas continentales: metodologias para su estudio. Argentina, Ed. Sur, Tomo I, 376p.

Brower, J.E. \& H.J. ZAR. 1984. Field \& Laboratory methods for general ecology. Dubuque, W.C. Brown Plub., $2^{\text {nd }}$ ed., 226p.

Hauer, F.R. \& V.H. Resh. 1996. Benthic macroinvertebrates, p. 339-369. In: F.R. HAUER \& G.A. LAMBERTI (Eds). Stream ecology. San Diego, Academic Press, 674p.

Higuti, J.; A.M. Takeda \& A.C. PAggi. 1993. Distribuição espacial das larvas de Chironomidae (Insecta, Diptera) do Rio Baía (MS-Brasil). Revista Unimar, Maringá, 15: 65-81.

Hynes, H.B. 1970. The ecology of running waters. Canada, University of Toronto Press, 555p.

Huamantinco, A.A. \& J.L. Nessimian. 1999. Estrutura e distribuição espacial da comunidade de larvas de Trichoptera (Insecta) em um tributário de primeira ordem do Rio Paquequer, Teresópolis, RJ. Acta Limnologica Brasiliensia, Botucatu, 11 (2): 1-16.

Revista Brasileira de Zoologia 22 (3): 613-618, setembro 2005 
KikUCHI, R.M. \& V.S. Uieda. 1998. Composição da comunidade de invertebrados de um ambiente lótico tropical e sua variação espacial e temporal, p. 157-173. In: J.L. Nessimian \& A.L. Carvalho (Eds). Ecologia de insetos aquáticos. Rio de Janeiro, PPGE-UFRJ, Séries Oecologia Brasiliensis, 309p.

KleEREKoper, H. 1990. Introdução ao estudo da limnologia. Porto Alegre, DNPA, $2^{\text {a }}$ ed., 329p.

LAKE, P.S. 1990. Disturbing hard and soft bottom communities: a comparison of marine and freshwater environments. Australian Journal of Ecology, Carlton, 15: 477-488.

LAMBERTI, G.A. \& V.H. ReSH. 1985. Comparatibility of introducted tiles and natural substrates for sampling lotic bacteria, algal and macroinvertebrates. Freshwater Biology, Oxford, 15: 21-30.

Lopretto, E.C. \& G. Tell. 1995. Ecosistemas de aguas continentals: metodologias para su studio. Argentina, Ed. Sur. Tomo III, $1397 \mathrm{p}$.

Mason JR., W.T. \& P.P. Yevich. 1967. The use of Phloxine B and Rose Bengal stains to facilite sorting benthic samples. Transactions of the American Microscopical Society, Lawrence, 86: 221-223.

Merritt, R.W. \& K.W. Cummins. 1996. An introduction to the aquatic insects of North America. Dubuque, Kendall/Hunt, $3^{\text {rd }}$ ed., 722p.

Nessimian, J.L. 1996. Comments on aquatic insect biodiversity from select localities in Rio de Janeiro State Brazil, p 255268. In: C.E.M. Bicudo \& N.A. Menezes.(Eds). Biodiversity in Brasil: a first approach. São Paulo, CNPq, 326p.

Nessimian, J.L.; R.M. Amorin; A.L. Henriques-Oliveira \& A.M. SanseVERINO. 2003. Chironomidae (Diptera) do Estado do Rio de Janeiro: levantamento dos gêneros e hábitats de ocorrência. Publicações Avulsas do Museu Nacional, Rio de Janeiro, 98: 1-16.

Oliveira, L.G.; P.C. Bispo \& N.C. Sá. 1997. Ecologia de comunidades de insetos bentônicos (Ephemeroptera, Plecoptera e
Trichoptera), em córregos do parque ecológico de Goiânia, Goiás, Brasil. Revista Brasileira de Zoologia, São Paulo, 14: 867-876.

PENNAK, R.W. 1978. Freshwater invertebrates of United States. New York, Jonh Wiley \& Sons, $2^{\text {nd }}$ ed., 803p.

Resh, V.H. \& D.M. Rosenberg. 1984. The ecology of aquatic insects. New York, Praeger Publishers, 625p.

Rincón, P.A. 1999. Uso do micro-habitat em peixes de riachos: métodos e perspectivas, p. 23-90. In: E.P. Caramaschi; R. MazzONI \& P.R. PERES-NeTO (Eds). Ecologia de peixes de riacho. Rio de Janeiro, PPGE-UFRJ, Série Oecologia Brasiliensis, vol. 4, 260p.

Rodríguez, S.E.; E. Bécares; F. Soto \& R. PACHO. 1998. Colonization of aquatic macroinvertebrates in a high mountain stream using artificial substrates. Verhandlungen Internationale Vereiningen Limnologie, Munich, 26: 1120-1124.

Sanseverino, A.M.; J.L. Nessimian \& A.L.H. Oliveira. 1998. A fauna de Chironomidae (Diptera) em diferentes biótopos aquáticos na Serra do Subaio (Teresópolis, RJ), p. 253-264. In: J.L. Nessimian \& A.L. Carvalho (Eds). Ecologia de insetos aquáticos. Rio de Janeiro, PPGE-UFRJ, Séries Oecologia Brasiliensis, 309p.

Serrano, M.A.S.; W. Severi \& V.J.S. Toledo. 1998. Comunidades de Chironomidae (Diptera) e outros macroinvertebrados em um rio tropical de planície - Rio Bento Gomes/MT, p. 265278. In: J.L. Nessimian \& A.L. Carvalho (Eds). Ecologia de insetos aquáticos. Rio de Janeiro, PPGE-UFRJ, Séries Oecologia Brasiliensis, 309p.

Statistica 5.1 for Windows. 1996. Tulsa, Computer program manual.

UIEDA, V.S. \& I.C.S.M. GAJARDO. 1996. Macroinvertebrados perifíticos encontrados em poções e corredeiras de um riacho. Naturalia, Belo Horizonte, 21: 31-47.

Whitton, B.A. 1975. River ecology. California, University of California Press, 725p.

Recebido em 14.X.2004; aceito em 01.VIII.2005. 